\title{
Características de pacientes com cárie severa da infância: análise de pacientes atendidos em centro de referência
}

\section{Characteristics of patients with severe childhood caries: analysis of patients assisted in a reference center}

\author{
Bruna Camargo* \\ Larissa Correa Pavinato** \\ Moisés Cardoso ${ }^{* * *}$ \\ Juliane Bervian ${ }^{* * * *}$ \\ Berenice Perussolo ${ }^{* * * *}$ \\ Eduardo Patussi ${ }^{* * * * * *}$
}

\section{Resumo}

Objetivo: verificar a correlação dos fatores comportamentais na ocorrência de cárie severa. Sujeitos e método: a amostra foi composta de 25 crianças de 6 a 71 meses, atendidas na Clínica Materno Infantil da Faculdade de Odontologia da Universidade de Passo Fundo. O estudo foi realizado em duas etapas: entrevista e exame clínico. A entrevista avaliou os hábitos alimentares e de higiene bucal, as variáveis demográficas e o acesso ao flúor. O exame clínico avaliou a presença de cárie severa (incluindo manchas brancas). Resultados: as principais características das crianças da amostra foram idade superior a 24 meses, menor escolaridade materna, prática da amamentação natural e uso da mamadeira, inclusive com sacarose, além daqueles indivíduos que não realizavam escovação noturna. Não houve diferença estatisticamente significativa na média de ceo-d para nenhum dos fatores em estudo. Conclusão: pode-se concluir que há a necessidade de formar profissionais voltados não apenas ao tratamento, mas também à prevenção das doenças na cavidade oral, como a cárie, mas, para que isso ocorra, é de extrema importância que se conheça o perfil das crianças que necessitam de maior cuidado.

Palavras-chave: Criança. Fatores de risco. Pré-escolar.

\section{Introdução}

A American Academy of Pediatric Dentistry (AAPD) classifica cárie precoce na infância (CPI) como a presença de um ou mais dentes decíduos cariados (lesões cavitadas ou não), perdidos (devido à cárie) ou restaurados antes dos 71 meses de idade. Para crianças de até 3 anos, qualquer sinal de cárie em superfície lisa é considerado severe early childhood caries - cárie severa na infância (CSI) ${ }^{1,2}$. Também é considerada CSI se, dos 3 aos 5 anos de idade, a criança apresentar mais de quatro, cinco e seis superfícies afetadas em dentes anteriores decíduos, aos 3, 4 e 5 anos de idade, respectivamente ${ }^{3}$. Este assunto, durante anos, persistiu inconsistente na literatura, tanto pelos limites do diagnóstico relativos ao desfecho "cavidade" e não ao processo da doença, quanto pela sua severidade ${ }^{4,5}$.

Para o desenvolvimento da cárie severa da infância, existem diversos fatores, tais como: hospedeiro susceptível, microbiota e dieta cariogênica. Esses fatores são influenciados por fatores secundários, tais como: hábitos de higiene bucal, acesso ao flúor, hábitos alimentares, fatores socioculturais e econômicos ${ }^{6}$. Claramente se reconhece que tais fa-

Pós-graduada em Ortodontia na Associação Gaúcha de Ortodontia, Rio Grande do Sul, Brasil.

Doutora em Odontologia. Professora da disciplina de Estágio em Clínica da Criança e do Adolescente na Faculdade de Odontologia da Universidade de Passo Fundo, Rio Grande do Sul, Brasil.

** Mestre em Odontologia. Doutorando na Universidade de Passo Fundo, Rio Grande do Sul, Brasil.

Doutora em Odontologia. Professora da disciplina de Estágio em Clínica da Criança e do Adolescente na Faculdade de Odontologia da Universidade de Passo Fundo, Rio Grande do Sul, Brasil.

***** Mestre em Odontologia. Professora da disciplina de Estágio em Clínica da Criança e do Adolescente na Faculdade de Odontologia da Universidade de Passo Fundo, Rio Grande do Sul, Brasil.

${ }^{* * * * *}$ Doutor em Odontologia. Professor da disciplina de Estágio em Clínica da Criança e do Adolescente na Faculdade de Odontologia da Universidade de Passo Fundo, Rio Grande do Sul, Brasil. 
tores agem de forma diferenciada na doença cárie dentária e nos primeiros anos de vida em função das peculiaridades inerentes a esta população $0^{5,7-9}$.

No Brasil, dados do levantamento de saúde bucal realizado em $2010^{10}$ revelaram que $27 \%$ das crianças de 18 a 36 meses apresentam no mínimo um dente com experiência de cárie, e a proporção chega a $45 \%$ em crianças de 5 anos de idade. Dessa forma, a cárie severa da infância é considerada um problema de saúde pública, pois atinge um grande número de crianças, gerando impacto negativo na qualidade de vida devido a problemas no desenvolvimento e no bem-estar dessas crianças, pois a cárie pode gerar dor, abscesso e infecção, o que impede a realização de uma alimentação adequada e afeta a autoestima, a sociabilidade e o bem-estar da crian$\mathrm{ça}^{11}$.

A cárie severa da infância, além de afetar a estrutura dental, pode influenciar negativamente no crescimento da criança, colaborando no retardo do crescimento e desenvolvimento. A explicação estaria no fato de que a dor gerada pela doença aumenta a produção de glicocorticoides, assim, em decorrência do sono perturbado, ocorre a diminuição da secreção do hormônio do crescimento ${ }^{12}$.

Diante do exposto, este estudo teve como objetivo avaliar os fatores comportamentais correlacionados ao desenvolvimento da doença cárie severa da infância, em pacientes atendidos na Clínica Materno Infantil da Faculdade de Odontologia da Universidade de Passo Fundo (UPF).

\section{Sujeitos e método}

O presente estudo é definido como observacional analítico transversal. A amostra de conveniência foi composta por crianças de ambos os gêneros, na faixa etária de 6 a 71 meses, atendidas na Clínica Materno Infantil da Faculdade de Odontologia da UPF, no período de março a junho de 2014. Os critérios de inclusão envolveram a assinatura do termo de consentimento livre e esclarecido pelos pais ou responsáveis, a presença de um responsável que apresentasse condições de responder a entrevista do estudo e a criança ser portadora de cárie severa da infância (desfecho) e consentir verbalmente com o exame clínico.

Este estudo foi aprovado pelo Comitê de Ética em Pesquisa da UPF (protocolo número 1.010.233). A coleta de dados foi realizada por uma equipe composta por uma entrevistadora e uma examinadora devidamente treinadas e calibradas. Dividiu-se a coleta em duas etapas: entrevista e exame clínico.

A entrevista foi realizada com os pais ou responsáveis, com questões referentes a variáveis demográficas (idade e gênero), hábitos de higiene bucal (idade de início do hábito e frequência de higieniza- ção), hábitos alimentares (presença de amamentação natural, idade do desmame, amamentação natural noturna, limpeza após amamentação natural noturna, uso da mamadeira, uso da mamadeira noturna, mamadeira contendo sacarose, limpeza após o uso de mamadeira noturna, frequência de consumo de sacarose na dieta e idade de introdução do açúcar na dieta) e acesso ao flúor (água fluoretada, dentifrício fluoretado).

O exame clínico para identificar a presença de cárie severa nas crianças foi realizado em equipo odontológico, utilizando o índice ceo-d ${ }^{13}$. Para a verificação da condição de cárie severa da infância, as lesões de manchas brancas também foram consideradas como cárie.

A análise dos dados foi feita com o software SPSS (versão 18.0). Foram descritas as variáveis quantitativas por média e desvio padrão e feita a comparação entre duas categorias dos fatores em estudo pelo teste $t$ de Student para amostras independentes e entre três ou mais categorias pelo teste de análise de variância (ANOVA). Foi considerado um nível de significância de $5 \%$.

\section{Resulltados}

Foram coletados dados de 25 crianças, 11 $(42,3 \%)$ do gênero masculino e $14(57,7 \%)$ do feminino. A média de idade foi de 4,2 anos ( $\mathrm{DP} \pm 1,0)$. As Tabelas 1 e 2 apresentam as características da amostra.

Tabela 1 - Características demográficas da amostra

\begin{tabular}{|c|c|c|c|}
\hline Variáveis demográficas & $\mathrm{N}$ & $\%$ & $\mathrm{P}$ \\
\hline \multirow{3}{*}{$\begin{array}{l}\text { 1. Gênero } \\
\text { Masculino } \\
\text { Feminino }\end{array}$} & & & \multirow[t]{3}{*}{0,6892} \\
\hline & 11 & 44,0 & \\
\hline & 14 & 56,0 & \\
\hline \multirow{4}{*}{$\begin{array}{l}\text { 2. Idade } \\
\begin{array}{l}1-2 \text { anos } \\
3-4 \text { anos } \\
5 \text { anos }\end{array}\end{array}$} & & & \multirow[t]{4}{*}{$<0,0001$} \\
\hline & 3 & 12,0 & \\
\hline & 19 & 76,0 & \\
\hline & 3 & 12,0 & \\
\hline \multirow{3}{*}{$\begin{array}{l}\text { 3. Família nuclear } \\
\text { Nuclear } \\
\text { Não nuclear }\end{array}$} & & & \multirow[t]{3}{*}{$<0,0001$} \\
\hline & 23 & 92,0 & \\
\hline & 2 & 8,0 & \\
\hline \multirow{3}{*}{$\begin{array}{l}\text { 4. Filho único } \\
\text { Sim } \\
\text { Não }\end{array}$} & & & \multirow[t]{3}{*}{0,2301} \\
\hline & 9 & 36,0 & \\
\hline & 16 & 64,0 & \\
\hline \multirow{7}{*}{$\begin{array}{l}\text { 5. Escolaridade materna } \\
1^{\circ} \text { grau incompleto } \\
1^{\circ} \text { grau completo } \\
2^{\circ} \text { grau incompleto } \\
2^{o} \text { grau completo } \\
\text { Ensino superior incompleto } \\
\text { Ensino superior completo }\end{array}$} & & & \multirow[t]{7}{*}{$<0,0001$} \\
\hline & 6 & 25,0 & \\
\hline & 6 & 25,0 & \\
\hline & 3 & 12,5 & \\
\hline & 7 & 29,2 & \\
\hline & 1 & 4,2 & \\
\hline & 1 & 4,2 & \\
\hline \multirow{4}{*}{$\begin{array}{l}\text { 6. Renda (R\$) } \\
600-1.000 \\
>1.000-2.500 \\
>2.500-3.600\end{array}$} & & & \multirow[t]{4}{*}{0,009} \\
\hline & 5 & 20,8 & \\
\hline & 15 & 62,5 & \\
\hline & 4 & 16,7 & \\
\hline
\end{tabular}

Fonte: autores. 
Tabela 2 - Características clínicas da amostra

\begin{tabular}{|c|c|c|c|}
\hline Variável & $\mathrm{N}$ & $\%$ & \\
\hline $\begin{array}{l}\text { 1. Valor ceo-d } \\
1-5 \\
6-10 \\
11-14 \\
\end{array}$ & $\begin{array}{c}10 \\
12 \\
3 \\
\end{array}$ & $\begin{array}{l}40,0 \\
48,0 \\
12,0\end{array}$ & 0,0686 \\
\hline $\begin{array}{l}\text { 2. Frequência de higiene bucal } \\
1 \text { vez ao dia } \\
\text { Mais de } 1 \text { vez ao dia }\end{array}$ & $\begin{array}{c}1 \\
24 \\
\end{array}$ & $\begin{array}{c}4,0 \\
96,0\end{array}$ & - \\
\hline $\begin{array}{l}\text { 3. Escovação antes de dormir }(\mathbf{n}=\mathbf{2 0}) \\
\text { Sim } \\
\text { Não }\end{array}$ & $\begin{array}{c}3 \\
17 \\
\end{array}$ & $\begin{array}{l}15,0 \\
85,0\end{array}$ & 0,0037 \\
\hline $\begin{array}{l}\text { 4. Início da higiene bucal } \\
0-5 \text { meses } \\
6-10 \text { meses } \\
11-14 \text { meses } \\
\end{array}$ & $\begin{array}{c}8 \\
12 \\
5\end{array}$ & $\begin{array}{l}32,0 \\
48,0 \\
20,0\end{array}$ & 0,2276 \\
\hline $\begin{array}{l}\text { 5. Uso de flúor } \\
\text { Sim } \\
\text { Não }\end{array}$ & $\begin{array}{c}24 \\
1 \\
\end{array}$ & $\begin{array}{c}96,0 \\
4,0 \\
\end{array}$ & - \\
\hline $\begin{array}{l}\text { 6. Amamentação natural }(\mathbf{n}=\mathbf{2 5}) \\
\text { Sim } \\
\text { Não }\end{array}$ & $\begin{array}{c}20 \\
5\end{array}$ & $\begin{array}{l}75,0 \\
25,0\end{array}$ & 0,0051 \\
\hline $\begin{array}{l}\text { 7. Tempo de amamentação natural }(\mathbf{n}=\mathbf{2 0}) \\
0-6 \text { meses } \\
7-11 \text { meses } \\
12-24 \text { meses } \\
\text { Acima de } 25 \text { meses }\end{array}$ & $\begin{array}{c}3 \\
2 \\
4 \\
11\end{array}$ & $\begin{array}{l}15,0 \\
10,0 \\
20,0 \\
55,0\end{array}$ & 0,2898 \\
\hline $\begin{array}{l}\text { 8. Aleitamento natural noturno }(\mathbf{n}=\mathbf{2 1}) \\
\text { Sim } \\
\text { Não }\end{array}$ & $\begin{array}{c}15 \\
6 \\
\end{array}$ & $\begin{array}{l}71,5 \\
28,5 \\
\end{array}$ & 0,0809 \\
\hline $\begin{array}{l}\text { 9. Uso de mamadeira }(\mathbf{n}=\mathbf{2 5}) \\
\text { Sim } \\
\text { Não }\end{array}$ & $\begin{array}{c}18 \\
7 \\
\end{array}$ & $\begin{array}{l}68,0 \\
32,0\end{array}$ & 0,0455 \\
\hline $\begin{array}{l}\text { 10. Mamadeira noturna }(\mathbf{n}=\mathbf{1 8}) \\
\text { Sim } \\
\text { Não }\end{array}$ & $\begin{array}{c}11 \\
7 \\
\end{array}$ & $\begin{array}{l}61,0 \\
39,0 \\
\end{array}$ & 0,4795 \\
\hline $\begin{array}{l}\text { 11. Mamadeira com sacarose }(\mathbf{n}=\mathbf{1 8}) \\
\text { Sim } \\
\text { Não }\end{array}$ & $\begin{array}{c}16 \\
2 \\
\end{array}$ & $\begin{array}{l}89,0 \\
11,0 \\
\end{array}$ & 0,0022 \\
\hline $\begin{array}{l}\text { 12. Frequência de sacarose }(\mathbf{n}=\mathbf{2 5}) \\
1 \text { vez ou menos por semana } \\
2 \text { a } 3 \text { vezes por semana } \\
\text { Até } 3 \text { vezes por dia } \\
\text { Mais de } 3 \text { vezes por dia }\end{array}$ & $\begin{array}{c}3 \\
10 \\
8 \\
4\end{array}$ & $\begin{array}{l}12,0 \\
40,0 \\
32,0 \\
16,0\end{array}$ & 0,1550 \\
\hline $\begin{array}{l}\text { 13. Introdução de sacarose }(\mathbf{n}=\mathbf{2 5}) \\
\text { Até os } 6 \text { meses } \\
\text { Entre } 7 \text { e } 11 \text { meses } \\
\text { Entre } 12 \text { e } 24 \text { meses } \\
\text { Após } 24 \text { meses }\end{array}$ & $\begin{array}{c}5 \\
8 \\
10 \\
2\end{array}$ & $\begin{array}{c}20,0 \\
32,0 \\
40,0 \\
8,0\end{array}$ & 0,1173 \\
\hline
\end{tabular}

Fonte: autores.

A Tabela 3 apresenta a comparação de médias de ceo-d entre as diferentes categorias dos fatores em estudo. Não houve diferença estatisticamente significativa na média de ceo-d para nenhum dos fatores em estudo.
Tabela 3 - Comparação da variável ceo-d entre as categorias dos fatores

\begin{tabular}{|c|c|c|c|}
\hline Variável & $\mathrm{n}^{*}$ & ceo-d & $\mathrm{P}$ \\
\hline $\begin{array}{l}\text { 1. Início higiene oral } \\
1-5 \\
6-10 \\
11-14\end{array}$ & $\begin{array}{c}9 \\
12 \\
5\end{array}$ & $\begin{array}{l}7,1 \pm 3,6 \\
6,2 \pm 4,3 \\
5,0 \pm 2,8\end{array}$ & 0,613 \\
\hline $\begin{array}{l}\text { 2. Frequência } \\
1 \text { vez ao dia } \\
\text { Mais de } 1 \text { vez ao dia }\end{array}$ & $\begin{array}{c}1 \\
25\end{array}$ & $\begin{array}{c}7,0 \\
6,2 \pm 3,8\end{array}$ & - \\
\hline $\begin{array}{l}\text { 3. Amamentação natural } \\
\text { Sim } \\
\text { Não }\end{array}$ & $\begin{array}{c}20 \\
1\end{array}$ & $\begin{array}{c}7,1 \pm 3,7 \\
2,0\end{array}$ & - \\
\hline $\begin{array}{l}\text { 4. Até que idade } \\
\text { Menos de } 6 \text { meses } \\
\text { De } 6 \text { a } 11 \text { meses } \\
\text { Entre } 1 \text { ano e } 2 \text { anos } \\
\text { Mais de } 2 \text { anos }\end{array}$ & $\begin{array}{c}3 \\
2 \\
4 \\
11\end{array}$ & $\begin{array}{l}7,7 \pm 6,0 \\
3,5 \pm 5,0 \\
8,5 \pm 5,3 \\
7,0 \pm 2,0\end{array}$ & 0,490 \\
\hline $\begin{array}{l}\text { 5. Aleitamento noturno } \\
\text { Sim } \\
\text { Não }\end{array}$ & $\begin{array}{c}15 \\
6\end{array}$ & $\begin{array}{l}6,4 \pm 3,0 \\
7,8 \pm 5,4\end{array}$ & 0,441 \\
\hline $\begin{array}{l}\text { 6. Limpeza } \\
\text { Sim } \\
\text { Não }\end{array}$ & $\begin{array}{c}3 \\
17\end{array}$ & $\begin{array}{l}9,3 \pm 2,5 \\
6,6 \pm 3,7\end{array}$ & 0,252 \\
\hline $\begin{array}{l}\text { 7. Mamadeira } \\
\text { Fez uso } \\
\text { Ainda faz } \\
\text { Nunca fez }\end{array}$ & $\begin{array}{c}7 \\
11 \\
8\end{array}$ & $\begin{array}{l}3,7 \pm 2,5 \\
6,6 \pm 3,8 \\
8,0 \pm 3,7\end{array}$ & 0,073 \\
\hline $\begin{array}{l}\text { 8. Mamadeira noturna } \\
\text { Sim } \\
\text { Não }\end{array}$ & $\begin{array}{l}11 \\
7\end{array}$ & $\begin{array}{l}4,5 \pm 3,2 \\
7,0 \pm 3,9\end{array}$ & 0,163 \\
\hline $\begin{array}{l}\text { 9. Açúcar na mamadeira } \\
\text { Sim } \\
\text { Não }\end{array}$ & $\begin{array}{c}16 \\
2\end{array}$ & $\begin{array}{l}5,8 \pm 3,5 \\
3,5 \pm 4,9\end{array}$ & 0,419 \\
\hline $\begin{array}{l}\text { 10. Limpeza de mamadeira } \\
\text { Sim } \\
\text { Não }\end{array}$ & $\begin{array}{c}13 \\
5\end{array}$ & $\begin{array}{l}5,0 \pm 3,2 \\
6,8 \pm 4,6\end{array}$ & 0,356 \\
\hline $\begin{array}{l}\text { 11. Frequência de açúcar } \\
1 \text { vez ou menos por semana } \\
2 \text { a } 3 \text { vezes por semana } \\
\text { Até } 3 \text { vezes ao dia } \\
\text { Mais de } 3 \text { vezes por dia }\end{array}$ & $\begin{array}{c}3 \\
10 \\
9 \\
4\end{array}$ & $\begin{array}{l}8,7 \pm 2,9 \\
5,1 \pm 4,1 \\
7,1 \pm 3,9 \\
5,5 \pm 2,6\end{array}$ & 0,438 \\
\hline $\begin{array}{l}\text { 12. Introdução } \\
\text { Antes dos } 6 \text { meses } \\
\text { Entre } 6 \text { e } 11 \text { meses } \\
\text { Entre } 1 \text { e } 2 \text { anos } \\
\text { Após } 2 \text { anos }\end{array}$ & $\begin{array}{c}5 \\
9 \\
10 \\
2\end{array}$ & $\begin{array}{l}7,8 \pm 4,3 \\
6,3 \pm 2,8 \\
5,1 \pm 3,4 \\
8,0 \pm 8,5\end{array}$ & 0,548 \\
\hline $\begin{array}{l}\text { 13. Flúor } \\
\text { Sim } \\
\text { Não }\end{array}$ & $\begin{array}{r}25 \\
1\end{array}$ & $\begin{array}{c}6,2 \pm 3,8 \\
9,0\end{array}$ & - \\
\hline
\end{tabular}

Valores de média \pm desvio padrão de ceo-d, utilizando teste t de Student para amostras independentes com duas categorias e teste de análise de variância (ANOVA) para variáveis com três categorias. Quando as caselas apresentaram um indivíduo, a comparação foi impossibilitada.

Fonte: autores. 


\section{Discussão}

A cárie severa na infância (CSI) substitui a nomenclatura anterior, "cárie de mamadeira", definida pela American Academy of Pediatric Dentistry $(\mathrm{AAPD})^{2}$ como qualquer sinal de superfície lisa cariada, sem ou com cavidade, em crianças menores de 3 anos de idade. Também pode ser considerada cárie severa na infância os casos em que uma criança de 3 anos apresentar quatro ou mais lesões de cárie, uma criança de 4 anos apresentar cinco ou mais lesões e uma criança aos 5 anos apresentar seis lesões cariosas ou mais.

Segundo Assed ${ }^{12}$ (2005), a cárie severa da infância, além de afetar a estrutura dental, pode influenciar negativamente o crescimento da criança, que passa a se alimentar de forma insatisfatória e, com isso, surgem inúmeras deficiências nutricionais que acarretam distúrbios do crescimento ${ }^{14}$.

Um trabalho realizado por Barros et al..$^{15}$ (2001) mostrou que a prevalência de cárie aumentou com a idade devido ao número crescente de superfícies dentárias expostas aos agentes etiológicos e à continuidade dos hábitos deletérios durante o tempo. Segundo alguns trabalhos ${ }^{16-18}$, a cárie não tratada apresenta um efeito cumulativo, aumentando sua prevalência à medida que a idade avança. O presente trabalho mostrou que a maior prevalência de CSI ocorreu em crianças com maior idade, reforçando esse efeito cumulativo.

Uma pesquisa realizada por Nelson-Filho e Asssed $^{19}$ (2005) mostrou que 88,23\% das crianças com cárie severa recebiam amamentação noturna e dessas $97,05 \%$ não realizavam higienização, dessa forma, os autores sugeriram que a amamentação noturna seguida da ausência de higiene oral são fatores de risco no desenvolvimento de cárie. $\mathrm{O}$ presente estudo também mostrou que os pacientes com CSI apresentaram alta prevalência no uso de mamadeiras noturnas (61\%) e na falta de higienização antes de dormir (85\%). De forma geral, dependendo da frequência, do conteúdo e do momento de consumo da mamadeira, em poucos meses, os dentes anteriores superiores podem passar da higidez a destruição coronária e perda dentária. Com base nas evidências disponíveis, sugere-se uma rede de causalidade para a ocorrência de cárie dentária que envolve fatores comportamentais: práticas alimentares e de higiene bucal, entre outros fatore ${ }^{20}$.

Declerck et al. ${ }^{21}$ (2008) verificaram que crianças cujas mães apresentaram baixo nível de instrução foram mais propensas a apresentar cárie severa da infância, pois estas fornecem líquidos açucarados em livre demanda aos filhos, inclusive durante a noite, e não oferecem auxílio ou incentivo para higienização bucal após a ingestão. Brandão e Heilborn ${ }^{22}$ (2006) mostram uma associação significativa entre a escolaridade materna e a cárie precoce. O presente estudo mostrou que 50\% das mães apresentavam menos de 8 anos de estudo e que $62,5 \%$ não apresentavam ensino médio completo. Em famílias cujo nível de instrução é maior, observam-se melhores cuidados com alimentação, higiene bucal e busca por informações de profissionais, a fim de prevenir o surgimento de doenças, como a cárie dentária ${ }^{21}$. O levantamento de dados mostrou que, a partir do momento em que o nível de escolaridade aumentou, o percentual de crianças com CSI diminuiu.

Alguns estudos ${ }^{11,12,17,19,22,23}$ mostraram a forte associação entre os fatores relacionados a dieta e higiene da criança com a presença de lesões cariosas e manifestação da cárie precoce da infância. No entanto, poucos trabalhos apresentam essas características relacionadas à cárie severa da infância. No presente estudo, dois terços da amostra apresentavam o hábito de utilizar mamadeira contendo sacarose e quase metade da amostra fazia uso da mamadeira durante a noite.

Estudos que observam a ocorrência da doença cárie necessitam evidenciar os fatores sociais e culturais relacionados aos agentes primários. Ao analisar o risco individual de cárie em crianças, faz-se necessário avaliar os dados relacionados ao seu convívio social e à sua inserção ambiental. Dessa forma, deve-se incluir dados dos responsáveis (principalmente da mãe) e do ambiente no qual a criança está inserida, pois alguns deles poderão interferir na saúde bucal da criança ${ }^{24}$. Este trabalho buscou coletar informações referentes à instrução dos responsáveis e à estrutura familiar, possibilitando observar a influência desses fatores na presença de CSI.

Algumas limitações deste trabalho devem ser observadas, como o número de participantes, que foi pouco expressivo e provavelmente levou à não detecção de diferença estatística entre a média ceo-d e os fatores em estudo. Além disso, trata-se de um estudo transversal, realizado em um centro de referência, o que impossibilita a extrapolação de dados diante das restrições metodológicas. Idealmente, seria necessário um estudo longitudinal, para então inferir sobre a causalidade.

No entanto, a caracterização de populações afetadas por uma doença pode ser importante para se traçar um perfil que necessite de maiores intervenções e de maiores cuidados. Este estudo mostrou que crianças com famílias de menor renda, com hábitos de higiene inadequados, que utilizam sacarose na mamadeira e cujas mães apresentam pouca instrução têm perfil característico de indivíduos com a doença em questão. Diante dessas observações, a equipe de saúde bucal pode criar estratégias para trabalhar com essas crianças com maior risco à cárie e com atividade da doença, a fim de diminuir a prevalência de cárie dentária na faixa etária de 0 a 5 anos ${ }^{25}$, considerando inclusive o fator preditor dos dentes decíduos para a saúde dos dentes permanentes ${ }^{26}$. 
Outro aspecto a ser destacado é que, na atualidade, já há consenso de que estratégias isoladas determinam apenas efeitos limitados a curto prazo, podendo ainda contribuir para o aumento das desigualdades em saúde ${ }^{27,28}$. Dessa forma, têm-se preconizado o desenvolvimento e a implementação de estratégias complementares para promover saúde que tenham demonstrado algum grau de efetivida$\mathrm{de}^{29}$. Nessa linha de argumentação, contribui o reconhecimento de que as doenças bucais apresentam fatores de risco em comum com outras doenças crônicas, reforçando a plausibilidade de intervenções integradas ${ }^{20,25,28}$.

\section{Conclusão}

Neste estudo, verifica-se como característica da amostra a prevalência de crianças com ausência de higiene oral antes de dormir, com tempo de aleitamento maior que 25 meses, além da alta frequência de aleitamento noturno e utilização da sacarose na dieta. No entanto, uma limitação do estudo é o pequeno número da amostra, comprometendo, dessa forma, os resultados estatísticos e a não correlação entre as variáveis do estudo. Contudo, salienta-se que, mesmo não podendo inferir sobre a correlação dos fatores comportamentais na ocorrência de cárie severa, neste estudo, são necessárias a formação de profissionais voltados à prevenção das doenças da cavidade oral e, sobretudo, a conscientização dos pais quanto aos cuidados, desde o primeiro ano de vida da criança, com higienização, aleitamento e dieta, a fim de reduzir a incidência da cárie dentária e manter o estado de saúde até a idade adulta.

\section{Abstract}

Objective: verify the correlation of behavioral factors on the occurrence of severe caries. Subjects and method: the sample consisted of 25 children aged six through 71 months, assisted at the Maternal and Child Clinic of the Faculty of Dentistry of the University of Passo Fundo, Brazil. The study was performed in two stages: interview and clinical examination. The interview evaluated food and oral hygiene habits, demographic variables, and access to fluoride. The clinical examination evaluated the presence of severe caries (including white spots). Results: the main characteristics of the children from the sample were age over 24 months, lower maternal level of education, breastfeeding, and bottle-feeding including sucrose, in addition to those who did not perform nighttime brushing. There was no statistically significant difference in the mean of ceo-d for any of the factors studied. Conclusion: it may be concluded that there is a need for training professionals who are focused on both treatment and prevention of diseases in the oral cavity, such as caries. However, to achieve this, it is extremely important to acknowledge the profile of children in need of extended care.

Keywords: Child. Risk factors. Preschool.

\section{Referências}

1. Drury TF, Horowitz AM, Ismael AI, Maertens MP, Rozier RG, Selwitz RH. Diagnosing and reporting early childhood caries for research purposes. A report of a work-sponsored by the National Institute of Dental and Craniofacial Research, the Health Resources and Services Administration, and the Health Care Financing Administration. J Public Health Dent 1999; 59(3):192-7.

2. American Academy of Pediatric Dentistry. Guideline Policy on Early Childhood Caries (ECC): classifications, consequences, and preventive strategies. Pediatr Dent 2015; $37(6): 50-2$

3. Raichert C, Gomes MAG, Barasuol JC, Ferreira FDM, Fraiz FC, Menezes JVNB. Cárie dentária e presença de placa visível em dentes ântero-superiores em crianças de 0 a 5 anos. Rev da APCD 2016; 70(1):38-43.

4. Pearce N. Traditional epidemiology, modern epidemiology, and public health. Am J Public Health 1996; 86(5):678-83.

5. Ismail AI, Sohn W. A systematic review of clinical diagnostic criteria of early childhood caries. J Public Health Dent 1999; 59(3):171-91.

6. da Silva MDGB, de Vasconcelos Catão MHC, de Andrade FJP, de Alencar CRB. Cárie precoce da infância: fatores de risco associados. Arch Health Invest 2017; 6(12):574-9.

7. Ismail AI. Prevention of early childhood caries. Community Dent Oral Epidemiol 1998; 26(1Suppl):49-61.

8. Feldens CA, Kramer PF, Sequeira MC, Rodrigues PH, Vitolo MR. Maternal education is an independent determinant of cariogenic feeding practices in the first year of life. Eur Arch Paediatr Dent 2012; 13(2):70-5.

9. Nunes VH, Perosa GB. Cárie dentária em crianças de 5 anos: fatores sociodemográficos, lócus de controle e atitudes parentais. Ciênc Saúde Coletiva 2017; 22, 191:200.

10. Brasil. Ministério da Saúde. Secretaria de Assistência à Saúde. Projeto SB Brasil 2010. Pesquisa Nacional de Saúde Bucal - resultados principais. Brasília: Ministério da Saúde; 2011.

11. Losso EM, Tavares MC, Silva JY, Urban Cde A. Severe early childhood caries: an integral approach. J Pediatr 2009; 85(4):295-300.

12. Assed S. Odontopediatria: bases científicas para a prática clínica. São Paulo: Artes Médicas; 2005.

13. World Health Organization. Oral health surveys: basic methods. 4. ed. Geneva: World Health Organization; 1997.

14. Feitosa S, Colares V, Pinkham J. The psychosocial effects of severe caries in 4-years-old children in Recife. Cad Saúde Pública 2006; 21(5):1550-6.

15. de Barros SG, Alves AC, Pugliese LS. Contribuição ao estudo da cárie dentária em crianças de 0-30 meses. Pesq Odontol Bras 2001; 15(3):215-22.

16. Maciel SSVV, Oliveira RLCC, Fernandes ACA, Steinhauser HC, Torres MJS, Freire MNB, et al. Prevalência da cárie precoce na infância em crianças de 6 a 36 meses em creches públicas de Caruaru/PE. Pesqui Bras Odontopediatria Clín Integr 2007; 7(1).

17. Feldens CA, Giugliani ER, Vigo Á, Vítolo MR. Early feeding practices and severe early childhood caries in four-year-old children from southern Brazil: a birth cohort study. Caries Res 2010; 44(5):445-52.

18. Assunção LRS, Vilella KD, Rocha DP, Menezes SL, Pinheiro RPS, Nascimento LS, et al. Epidemiologia da cárie dentária em crianças da primeira infância no município de Belém, PA. Rev APCD 2015; 69(1):74-9. 
19. Nelson-Filho N, Assed S. Cárie de mamadeira. In: Assed S. Odontopediatria: bases científicas para a prática clínica. São Paulo: Artes Médicas; 2005. p. 344-8.

20. Feldens CA, Kramer PF, Rodrigues PH, Bervian J. Fatores de risco na cárie dentária na infância. In: Feldens CA, Kramer PF. Cárie dentária na infância: uma abordagem contemporânea. São Paulo: Santos; 2013. p. 93-109.

21. Declerck D, Leroy R, Martens L, Lesaffre E, Garcia Zattera MJ, Broucke SV, et al. Factors associated with prevalence and severity of caries experience in preschool children. Comm Dent Oral Epidemiol 2008; 36(2):168-78.

22. Brandão ER, Heilborn ML. Sexualidade e gravidez na adolescência entre jovens de camadas médias do Rio de Janeiro, Brasil. Cad Saúde Pública 2006; 22(7):1421-30.

23. Dini EL, Holt RD, Bedi R. Caries and its association with infant feeding and oral health related behaviors in 3-4-yearold Brazilian children. Comm Dent Oral Epidemiol 2000; 28(4):241-8.

24. Fadel CB. Cárie dental precoce: qual o verdadeiro impacto da dieta em sua etiologia? Publ UEPG Cienc Biol Saúde 2003; 9(3/4):83-9.

25. Petersen PE. The World Oral Health Report 2003: continuous improvement of oral health in the 21 st century-the approach of the WHO Global Oral Health Programme. Comm Dent Oral Epidemiol 2003; 31(Supl 1):3-23.

26. Li Y, Wang W. Predicting caries in permanent teeth from caries in primary teeth: an eight-year cohort study. J Dent Res 2002; 81(8):561-6.

27. Schou L, Wight C. Does dental health education affect inequalities in dental health? Comm Dent Health 1994; 11(2):97-100.

28.' Sheiham A, Watt RG. The common risk fator approach: a rational basis for promoting oral health. Comm Dent Oral Epidemiol 2000; 28(6):399-406.

29. World Health Organization. The Ottawa Charter for Health Promotion. Geneva: World Health Organization; 1986.

\section{Endereço para correspondência:}

Juliane Bervian

BR 285, São José

99052-900, Passo Fundo, RS, Brasil

Telefone: (54) 9983-1864

E-mail: jbervian@upf.br

Recebido: 17/05/18. Aceito: 03/07/ 18. 